in acute than chronic schizophrenics (Dalen, 1975; Pulver et al, 1983). Even more telling, three investigations have found that the risk-increasing effect of influenza epidemics is more readily detected in female than male schizophrenics (Mednick et al, 1991; O'Callaghan et al, 1991; Takei et al, 1992).

How can we reconcile these seeming inconsistencies? We stand by the view that early-onset schizophrenia is a consequence of deviant brain development, and that, like other neurodevelopmental disorders, it is commoner in males. We have suggested elsewhere that it may result from an aberration in the genetic control of early brain growth or obstetric complications, or some combination of the two (Jones \& Murray, 1991).

It remains uncertain whether the 'schizophrenogenic' effect of prenatal exposure to influenza operates preferentially in females, or whether it is simply easier to demonstrate this effect in female schizophrenics because of the smaller numbers of cases with a different aetiology. On the other hand, one can make a provisional case from the evidence reviewed above that prenatal exposure to influenza epidemics is more likely to be aetiologically implicated in later-onset milder schizophrenia, which is commoner in females. This form is associated with less premorbid dysfunction and gross structural abnormalities than the type which we have, perhaps confusingly, called the 'neurodevelopmental' type, but on such reasoning, would also have its origins very early in life. This tentative hypothesis is compatible with Castle's own finding that when schizophrenia is subtyped into 'neurodevelopmental' and later-onset forms, it is the later-onset paranoid form which shows a winter-birth excess. The decline in epidemic influenza and its complications since the 1930 s is also a possible explanation for the report of Waddington \& Youssef (1992) that the recent decline in rates of schizophrenia is greatest in later-onset female cases.

Such a simple heterogeneity model of schizophrenia is unlikely to be completely correct, and does not even begin to resolve the relative contributions of heredity to the different forms. However, it has the merit of being testable, and we shall look forward to Castle \& Gill, among others, examining its strengths and weaknesses.

Boyd, J. H., Pulver, A. E. \& Stewart, W. (1986) Season of birth: schizophrenia and bipolar disorder. Schizophrenia Bulletin, 12, 173-185.

DALEN, P. (1975) Season of Birth: A Study of Schizophrenia and other Mental Disorders. Amsterdam: Elsevier.

Jones, P. \& MURRAY, R. M. (1991) The genetics of schizophrenia is the genetics of neurodevelopment. British Journal of Psychiatry, $158,615-623$.
Mednick, S. A., Machon, R. A., Huttunen, M., et al (1990) Influenza and schizophrenia: Helsinki vs Edinburgh. Archives of General Psychiatry, 47, 875-876.

Murray, R. M. \& O'Callaghan, E. (1991) The congenital and adult-onset psychoses: Kraepelin lost and Kraepelin found. In Concepts of Mental Disorder (eds A. Kerr and H. McClelland), pp. 48-65. London: Gaskell.

O'Callaghan, E., Sham, P., Take, N., et al (1991) Schizophreniz after prenatal exposure to 1957 influenza epidemic. Lancet, 337, 1248-1250.

Pulver, A. E., Stewart, W., Carpenter, W. T., et al (1983) Risk factors in schizophrenia. British Journal of Psychiatry, 143, 389-396.

Take, N., O'Callaghan, E., Sham, P., et al (1992) Winter birth excess in schizophrenia: its relationship to place of birth. Schizophrenia Research, 6, 102.

WADDINGTON, J. L. \& Yousser, H. A. (1992) The declining incidence of schizophrenia in a rural Irish population of unusual homogeneity: secular trend towards early onset and male predominance. Schizophrenia Research, 6, 101.

P. C. SHAM

E. O'Callaghan

N. TAKEI

R. M. MURRAY

Department of Psychological Medicine

Institute of Psychiatry

De Crespigny Park

London SE5 8 AF

\section{Influenza and schizophrenia in Japan}

SiR: Sham et al (Journal, April 1992, 160, 461-466) show an effect of influenza on pre-schizophrenics in utero. This paper is a more sophisticated replication of the study by Barr et al (1990) showing a correlation between prenatal exposure to influenza epidemics and an increase of schizophrenic births in a longitudinal sample.

Although six studies have examined schizophrenic births following the 1957 influenza A2 pandemic, the results have not yet converged. Three studies, from Sweden (Mednick et al, 1988) and England (O'Callaghan et al, 1991; Fahy et al, 1992), demonstrated positive findings but two others, from Northwick Park (Crow et al, 1992) and the USA (Torrey et al, 1992), did not. The final study, from Scotland (Kendell \& Kemp, 1989) is equivocal. This inconsistency requires more studies to clarify the aetiological role of influenza on schizophrenia.

In Japan, an increase in the reported number of deaths attributable to influenza epidemic began in June 1957, reaching a peak in July and a further greater peak in December (Statistics of Communicable disease and Food Poisoning, 1957/58: Ministry of Health and Welfare, Japan). We obtained information on all dates of birth of individuals born between 1955 and 1960 , who were treated for schizophrenia during the study period, November to December 1991, at 12 mental hospitals around the 
Tokyo metropolitan areas. The data were provided by clinical psychiatrists who were blind to the study hypothesis. Hospital diagnosis was used. We defined the index year from August 1957, following the very first wave of the epidemic, to July 1958. We compared the number of schizophrenic births in each month of the index year with the average number of births in the corresponding month of the two years before, and following, the index year.

There were 147 schizophrenics born during the index year and 689 in the four control years. The distribution of birth rates in the index year $(1957 / 58)$ was compared with that of the average birth rates over the four control years. A $\chi^{2}$ test for comparison of distributions of schizophrenic births between these index and control groups yielded 10.50 on 11 d.f. (degrees of freedom), indicating an overall non-significant difference. However, when adjusted residuals (ARs) (Haberman, 1973) were examined, the observed number of births $(n=20)$ in April 1958, four months after the peak influenza deaths, was found to be unusually high $(A R s=2.253, P<0.012)$ compared with the average number of births $(n=12.50)$ over the four control years. Calculation of the ratios of affected births in each month of the index year to the average number of births in the corresponding month in the control years revealed that the risk of later developing schizophrenia was elevated by $60 \%$ in those exposed to the peak of the epidemic, four months before birth.

Although the present study is limited by the relatively small sample size, our results support the reports of O'Callaghan et al (1991) and Mednick et al (1988). Furthermore, the fact that the ratio of general population births in April 1958 to the average of population births in the four control years is 0.995 , suggests that the excess of schizophrenic births cannot be explained by a changing birth pattern in the general population. The period four months before birth corresponds roughly to the 5th-6th month of gestation. However, taking into account the time lag between the peak of prevalence of, and that of death rates from, influenza, the susceptible period appears to be between the 3rd and 6th month of gestation. This study is therefore compatible with the view that maternal exposure to influenza in the second trimester increases the risk of later schizophrenia in the foetus.

Barr, C. E., Mednick, S. A. \& Munk-Jorgensen, P. (1990) Exposure to influenza epidemics during gestation and adult schizophrenia: a 40-year study. Archives of General Psychiatry, 47, 869-874.

Crow, T. J., Done, D. J. \& Johnstone, E. C. (1992) Schizophrenia is not due to maternal influenza in the second (or other) trimester of pregnancy. Schizophrenia Research, 6, 99-100
Fahy, T. A., Jones, P. B., Sham, P. C., el al (1992) Schizophrenia in afro-Caribbeans in the UK following prenatal exposure to the 1957 A2 influenza epidemic. Schizophrenia Research, 6, 98-99.

Haberman, S. J. (1973) The analysis of residuals in cross-classified tables. Biometrics, 29, 205-220.

Kendell, R. E. \& KEMP, I. W. (1989) Maternal influenza in the etiology of schizophrenia. Archives of General Psychiatry. 46, 878-882.

Mednick, S. A., Machon, R. A., Huttenen, M. O., el al (1988) Adult schizophrenia following prenatal exposure to an influenza epidemic. Archives of General Psychiatry, 45, 189-192.

O' Callaghan, E., Sham, P. C., Takei, N., et al (1991) Schizophrenia after prenatal exposure to 1957 A2 influenza epidemic. Lancet, 337, 1248-1250.

Torrey, E. F., Bowler, A. E. \& RAwlings, R. (1992) Schizophrenia and the 1957 influenza epidemic. Schizophrenia Research. 6, 100

H. KUNUGI

S. NANKO

Department of Psychiatry

Teikyo University School of Medicine

Kaga

Itabashi-Ku

Tokyo 173

Genetics Department

Institute of Psychiatry

Denmark Hill

London SE5 8AF

Schizophrenia following prenatal exposure to influenza epidemics between 1939 and 1960

SIR: The correlation between maternal viral infection and the later occurrence of increased incidence of schizophrenia appears initially to be most convincing. The study of Sham et al (Journal, April 1992, 160, 461-466) uses influenza deaths to identify epidemics occurring at what are hypothesised to be vulnerable gestational periods of development.

Another hypothesis worthy of consideration is that children who are born during an influenza epidemic are more likely to have lost a grandparent in the pre- or post-natal period due to the influenza itself. In 1978, Walsh investigated the concurrence of grandparent death and the birth of an offspring who subsequently developed a schizophrenic illness. She found that of a sample of 70 schizophrenic patients, $41 \%$ experienced grandparental death within two years before or following a birth. In contrast, this concurrence of events was found in only $20 \%$ of families in a disturbed, non-schizophrenic group and in only $8 \%$ of cases in a normal control group.

The study is but one of several that suggest that, in mourning, a bereaved parent may be emotionally unavailable to spouse and infant at critical stages of development or that the process of mourning itself may be blocked and the child may assume a special 